\title{
MESFET GaAs à grille interrompue. Analyse du fonctionnement pour la photo-détection
}

\author{
D. Pascal, P. Dansas, C. Bru et S. Laval \\ Institut d'Electronique Fondamentale, Université Paris-Sud, \\ CNRS UA 22, Bâtiment 220, 91405 Orsay Cedex, France
}

(Reçu le 23 janvier 1987, accepté le 5 mars 1987)

\begin{abstract}
Résumé. - Une nouvelle structure de FET dans laquelle la grille est interrompue sur une certaine distance est décrite et son fonctionnement en tant que photo-détecteur est analysé. On montre comment les résistances d'accès sont modulées en obscurité par la tension grille en régime quasi continu. La réponse à un éclairement quasi stationnaire est étudiée : elle est maximale quand le canal est complètement pincé. La réponse à un éclairement impulsionnel picoseconde présente deux composantes : l'une, rapide, de photo-diode, l'autre, plus lente, de photo-résistance.

Abstract. - A modified OPFET structure, in which a gap is made in the middle of gate strip, has been used as a photo-detector. In darkness, the value of the access resistances has been found to be dependent on the bias voltage applied to the two half gates. A maximum photoresponse of the device to long light pulses is observed as the channel is pinched-off. The response to picosecond light pulses has two components : a « photodiodelike » one and a "photo-resistance-like » one (the faster and slower one respectively).
\end{abstract}

\section{Introduction.}

Les transistors MESFET en GaAs ont été largement utilisés pour la détection optique très rapide par de nombreux auteurs [1,2]. Différentes composantes du photo-courant drain-source ont été mises en évidence [3]. Ces transistors sont habituellement éclairés par le faisceau à détecter, dans l'espace drain-source, ou comportent une grille semi-transparente.

Nous présentons ici une étude expérimentale sur une structure de FET originale dans laquelle la grille est interrompue sur une certaine distance et dont les deux moitiés ainsi définies, sont reliées ensemble, par ailleurs, à un même potentiel. En éclairant au niveau de l'interruption de grille, les photons incidents créent des porteurs directement dans la zone à fort champ.

La structure est représentée sur la figure 1. Notons l'extrême étroitesse du canal : $3 \mu \mathrm{m}$, et l'importance relative des résistances d'accès : la longueur de grille est de $0,8 \mu \mathrm{m}$ pour une distance drain-source de $4 \mu \mathrm{m}$. Pour une tension $V_{\text {gs }}$ égale à 0 , si l'on admet que le potentiel de surface est voisin de la hauteur de la barrière Schottky, la zone désertée est d'épaisseur uniforme, et les résistances d'accès sont les $4 / 5$ de la résistance totale drain-source.

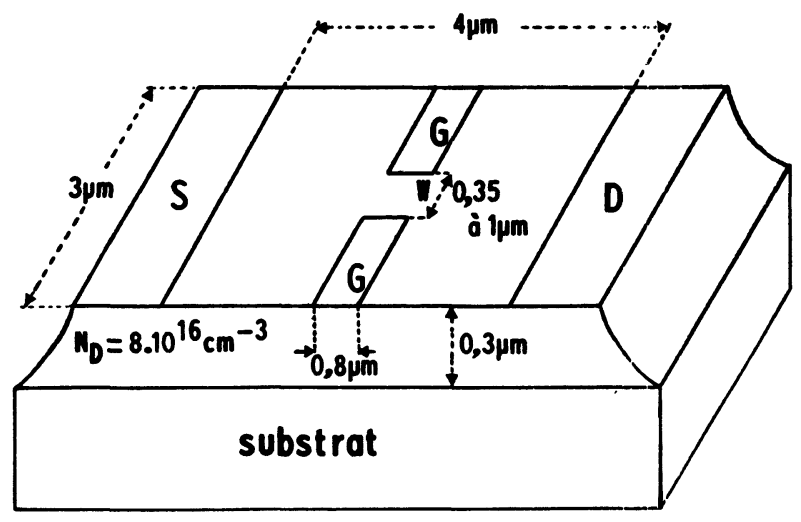

Fig. 1. - Schéma du transistor.

[Schematic view of the transistor.]

\section{Caractéristiques d'obscurité.}

Pour une interruption de grille de $1 \mu \mathrm{m}$, les $2 / 3$ seulement du canal sont commandés par la grille ; si l'on applique une tension $V_{\mathrm{T}}$ sur la grille (c'est-àdire suffisante pour déserter toute la couche active), le courant drain est divisé approximativement par 3, à condition que la tension grille soit appliquée suffisamment rapidement (en un temps inférieur à $10 \mu$ s environ). Si la tension grille est maintenue plus 
longtemps (typiquement $100 \mathrm{~ms}$ ) le courant drain continue de décroître, et l'espace inter-grille se pince', comme si l'interruption de grille était beaucoup plus courte. Ainsi, en statique, le potentiel de surface varie avec la polarisation de grille comme cela a été reconnu dans des articles récents [4-6].

Une conséquence de cette variation est le pincement des zones d'accès, du moins, en statique. En effet, une polarisation grille impulsionnelle (pendant $5 \mu$ s par exemple) est trop rapide pour faire varier le potentiel de surface. La caractéristique $I_{\mathrm{D}}\left(V_{\mathrm{gs}}\right)$ tracée dans ces conditions, permet de calculer la résistance d'accès $R_{\mathrm{a}}$ qu'on trouve égale à peu près aux $4 / 5$ de la résistance totale pour $V_{\mathrm{gs}}=0$ $\left(R_{\mathrm{a}} \simeq 950 \Omega\right)$, [7]. Par contre, une polarisation continue négative de grille conduit à une résistance d'accès effective bien plus faible : $R_{\mathrm{a}} \simeq 750 \Omega$ (la résistance d'accès géométrique étant partiellement commandée par la grille). Le canal peut être pincé complètement y compris dans l'espace d'interruption de la grille, en appliquant une tension $V_{\mathrm{gs}}$ suffisante, d'autant plus grande que l'interruption de grille est plus large. Voir figure 2.

\section{Eclairement quasi stationnaire.}

$\mathrm{Vu}$ l'extrême petitesse du dispositif, un système optique performant est utilisé pour l'éclairer sur une
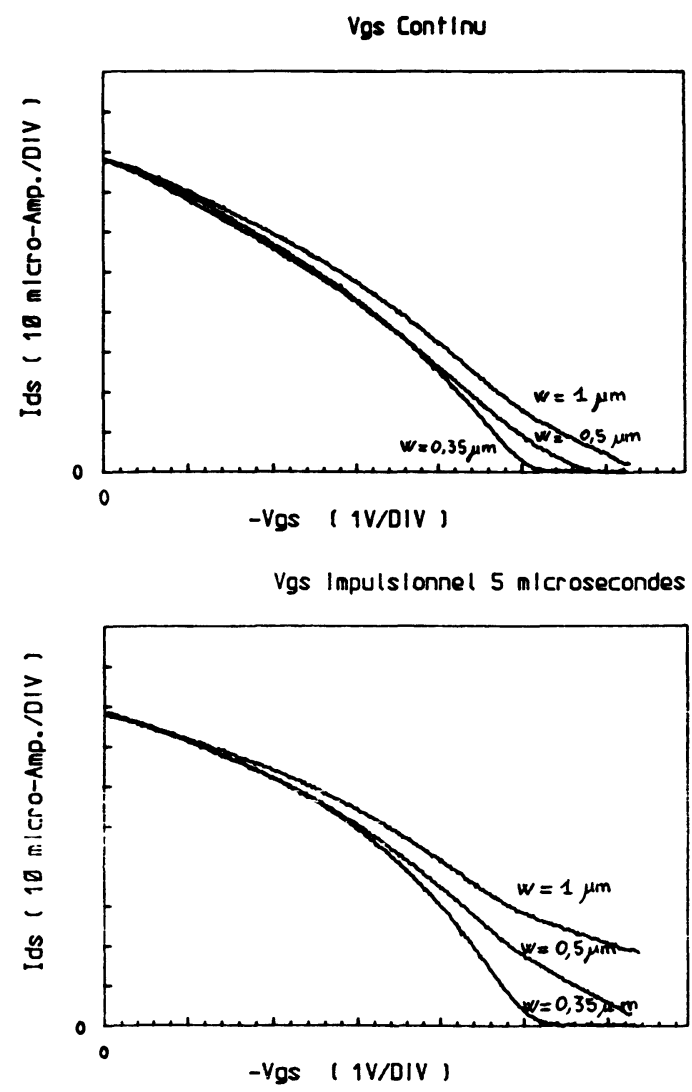

Fig. 2. - Caractéristiques d'obscurité $I_{\mathrm{ds}}\left(V_{\mathrm{gs}}\right)$.

$\left[I_{\mathrm{ds}}\left(V_{\mathrm{gs}}\right)\right.$ characteristics. $]$ toute petite surface (objectif de microscope d'ouverture numérique 0,6 , précédé d'un filtre spatial). Une tache d'environ $1 \mu \mathrm{m}$ de diamètre mesurée à $1 / e^{2}$ peut être centrée sur la zone d'interruption de la grille à $0,1 \mu \mathrm{m}$ près. L'éclairement quasi stationnaire est obtenu à l'aide d'un laser $\mathrm{HeNe}$ $(\lambda=0,6328 \mu \mathrm{m})$ dont le faisceau est découpé par un hacheur mécanique. Le temps d'éclairement peut être ajusté de $5 \mu \mathrm{s}$ à $1 \mathrm{~ms}$, et l'intensité du faisceau, de quelques dizaines de femtowatt à quelques dizaines de nanowatt. Quand on augmente la polarisation inverse de grille, on constate un accroissement du photo-courant drain-source qui présente un maximum pour le pincement complet. (Voir Fig. 3). Pour $V_{\mathrm{gs}}=0$, le photo-courant est sensiblement indépendant de la largeur de l'interruption : c'est le photocourant d'une photo-résistance de $4 \mu \mathrm{m}$ de distance inter-électrode et de $3 \mu \mathrm{m}$ de large. Malgré la petitesse de la tache, la totalité de la surface entre drain et source reçoit un éclairement de répartition sensiblement gaussienne.

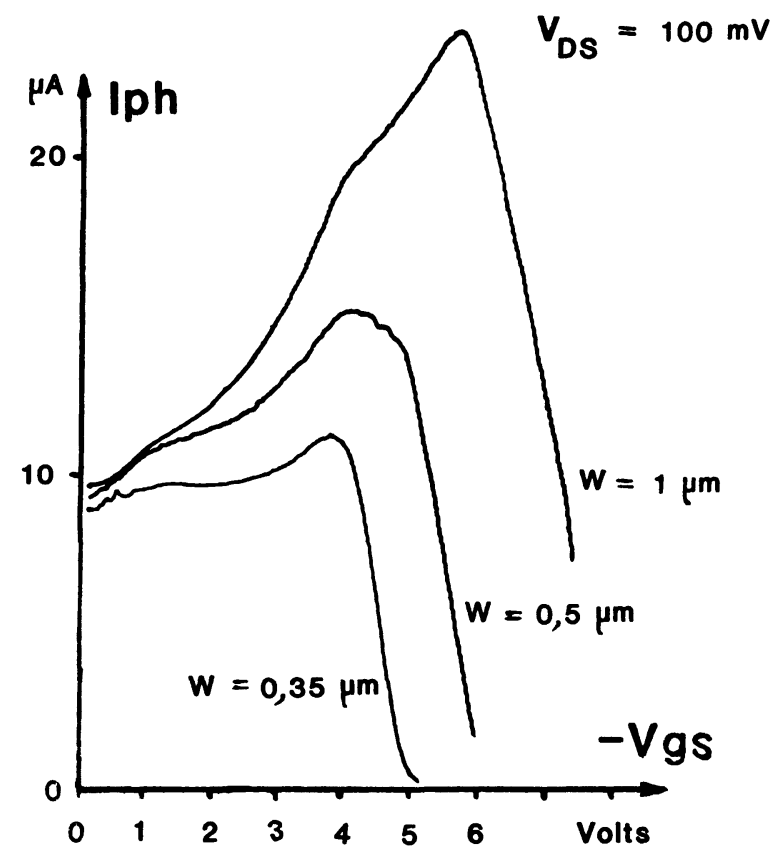

Fig. 3. - Photocourant en fonction de $V_{\mathrm{gs}}$ pour différentes largeurs d'interruption.

[Photocurrent versus $V_{\mathrm{gs}}$ for several values of $W$.]

Au fur et à mesure qu'on pince le canal, le courant drain total diminue, la chute de tension dans les résistances d'accès diminue et la tension appliquée $V_{\text {DS }}$ se retrouve aux bornes du canal: à $V_{\text {DS }}$ constant, le champ électrique augmente donc. Plus l'interruption est large, plus le photo-courant est grand et plus la tension $V_{g s}$ doit être grande pour qu'il atteigne son maximum. On a là, une photorésistance dont on peut contrôler le courant d'obscurité, et l'annuler pratiquement. Le gain de cette 
photo-résistance (rapport du nombre d'électrons circulant dans la structure sur le nombre de photons incidents) est régi par les pièges de surface et par l'éclairement. Il est égal au rapport de la durée de vie (qui est d'autant plus grande que l'éclairement est plus faible) sur le temps de transit [8]. Les parties faiblement éclairées participent donc relativement davantage au photo-courant. Les gains mesurés vont de $10^{4}$ à $10^{6}$. Par contre, un éventuel photo-courant de grille ne bénéficie pas de ce gain : les porteurs photo-créés dans la zone de charge d'espace sont collectés par la grille et ne sont pas réinjectés, le contact étant bloquant. Le photo-courant grille est donc très faible et n'induit pas de variation du courant drain via la résistance de source et la pente $\mathrm{du}$ transistor (qui est d'ailleurs très faible, vu la largeur du canal actif : $\left.g_{\mathrm{m}}=0,3 \mathrm{~mA} / \mathrm{V}\right)$.

\section{Eclairement impulsionnel.}

L'étude du comportement de ce phototransistor en impulsions lumineuses ultra-courtes, a été faite à l'aide d'un laser à colorant (Rhodamine 6G) pompé en synchronisme par un laser à Argon ionisé à modes bloqués en phase. La longueur d'onde est voisine de $0,6 \mu \mathrm{m}$ et la durée des impulsions voisine de 7 ps. La mesure du photocourant est faite par un oscilloscope à échantillonnage de $50 \Omega$ d'impédance d'entrée. Les temps de montée mesurés les plus courts sont d'environ 50 ps. Les valeurs crêtes des

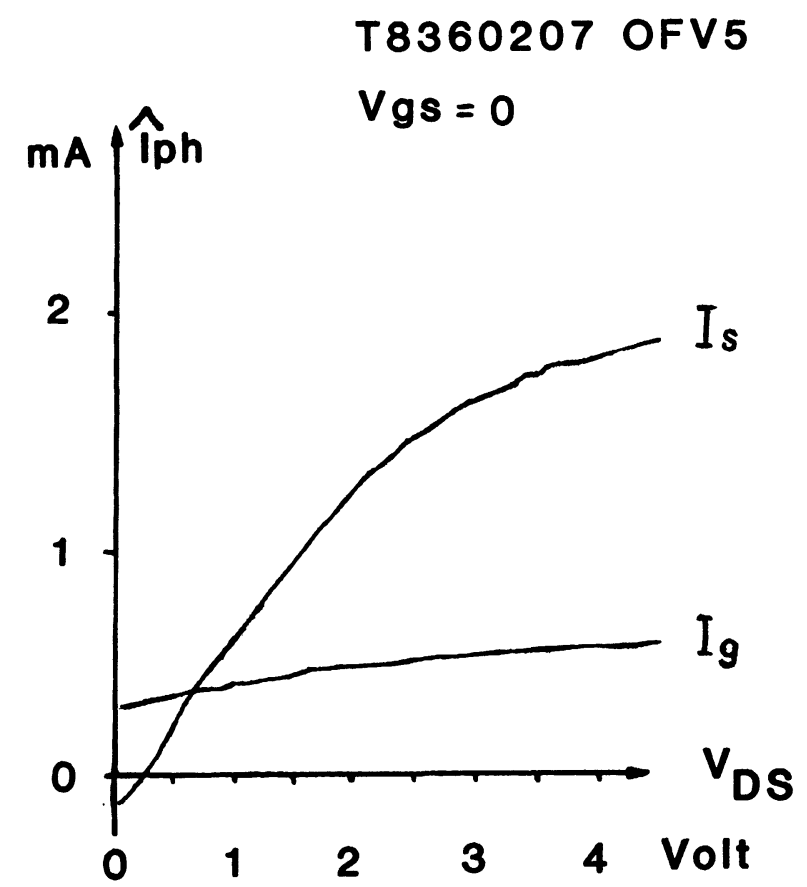

Fig. 4. - Valeurs crêtes du photocourant grille et drainsource en fonction de $V_{\mathrm{ds}}$.

[Peak values of gate and channel photocurrents versus $V_{\text {ds. }}$ ] courants sont typiquement mesurées au bout de 80 à 100 ps. Ces temps de montée sont limités par l'appareillage et les éléments parasites du support du boîtier du transistor.

Si l'on suppose qu'au bout d'un temps aussi court les mécanismes de recombinaison et de piégeage n'ont pas encore eu le temps de modifier les populations initiales, le photocourant sera proportionnel au nombre de photons incidents, tout étant identique par ailleurs. C'est ce que l'expérience confirme à peu près. La photodiode Schottky délivre maintenant un courant grille du même ordre de grandeur que le courant drain. Par exemple, pour $V_{\text {DS }}=0$, c'est le courant photovoltaïque qui prend naissance dans la zone de charge d'espace sous la grille qui est mesuré dans le circuit de grille et qui est dérivé approximativement par moitié vers la source

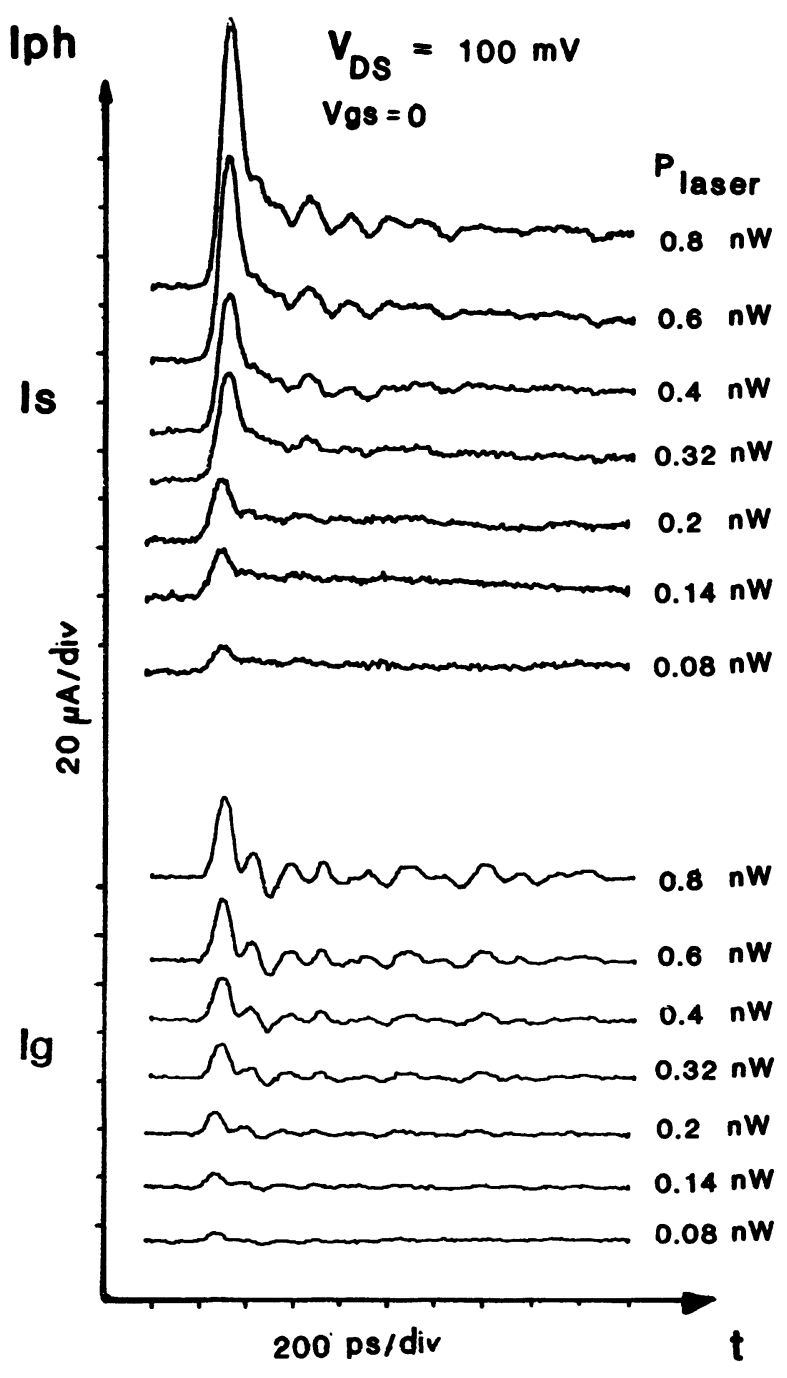

Fig. 5. - Réponse temporelle du dispositif pour différentes intensités lumineuses. $I_{\mathrm{s}}$ : Photocourant source. $I_{\mathrm{g}}$ : Photocourant grille.

[Photocurrent versus time for several light intensities. $I_{\mathrm{s}}$ : Channel photocurrent. $I_{\mathrm{g}}$ : Gate photocurrent.] 
et vers le drain, si les résistances d'accès sont à peu près égales. C'est ce qu'on peut voir sur la figure 4 .

Quand on augmente $V_{\mathrm{DS}}$, on obtient un photocourant $I_{\mathrm{DSph}}$ qui croît avec $V_{\mathrm{DS}}$, et qui s'ajoute à la composante photovoltaïque. On peut alors distinguer dans la réponse temporelle (Fig. 5), une composante rapide (temps de montée et de descente inférieurs à 100 ps) et une traîne lente (d'autant plus importante relativement que l'éclairement est plus faible). Les mécanismes de recombinaison et de piégeage dans les zones d'accès et, éventuellement, à l'interface avec le substrat, semblent responsables de cette longue constante de temps. Des expériences sont en cours (notamment en choisissant différentes résistances de grille) pour mettre en évidence l'effet transistor : la modulation du courant drain par la tension $V_{\mathrm{gs}}$ induite par le courant grille à travers la résistance de grille [3].

\section{Conclusion.}

Une nouvelle structure pour la photodétection a été présentée : elle se comporte comme un MESFET classique : le pincement total du canal est possible ; d'autre part, l'interruption de grille permet aux photons incidents de créer des paires électrons-trous dans la région de champ fort.

\section{Remerciements.}

Nous remercions MM. C. Arnodo et E. H. Perea de la Thomson-CSF à Corbeville pour l'élaboration des échantillons.

\section{Bibliographie}

[1] Gammel, J. C., Ballantyne, J. M., IEEE IEDM Tech. Dig. (1978) 120.

[2] Sugeta, T. and Mizushima, Y., Jpn J. App. Phys. 19 (1980) 27.

[3] UmedA, T. and Chо, Y., Jpn J. App. Phys. 24 (1985) L367.

[4] Wallis, R. H., Faucher, A., Pons, D. and Jay, P. R., Proc. of Int. Symp. GaAs and Related Compounds, Biarritz, 1984.
[5] Blight, S. R., Wallis, R. H., Thomas, H., Electron. Lett. 22 (1986) 47.

[6] Blight, S. R., Wallis, R. H., Thomas, H., IEEE Trans. Electron Devices ED-33 (1986) 1447.

[7] FukuI, H., Bell. Syst. Tech. J. (1979) 711.

[8] Vilcot, J. P., Vaterkowski, J. L., Decoster, D., Electron. Lett. 20 (1984) 86. 